\title{
A Reliability Evaluation Method of Power Information Communication Network
}

\author{
Yu Song ${ }^{1, a}$, Jianghong Shi ${ }^{1, b}$ \\ ${ }^{1}$ School of Control and Computer Engineering, North China Electric Power University, Baoding \\ 071003, China \\ aemail: syu1999@163.com, bemail:766227399@qq.com
}

\author{
Keywords: Cyber-Physical System; Power Information Communication Network; Reliability \\ Evaluation; Factor Analysis
}

\begin{abstract}
Along with the unceasing infiltration and fusion of power cyber space and physical space, a typical power cyber physical complex system is constructed, which brings new challenges to the reliability evaluation of power information communication network. For the reliability assessment of power information communication network is a multi index issue where the indexes have deep dependence and coupling, a comprehensive reliability evaluation model is constructed. At first, this paper introduces the related theory of factor analysis, and then constructs the reliability evaluation model based on factor analysis. Finally, the effectiveness of the proposed method is shown by applying this method to an example of a power communication network.
\end{abstract}

\section{Introduction}

Power communication network is an important infrastructure of power system, which is the basis of power network dispatching automation, network operation and management modernization. It is an important means to ensure the security, stability and economic operation of power network. Especially with the development of power system information and intelligence, the CPS feature is more and more obvious [1]. The formation of CPS makes the original huge system become more complex, which is called "a system in the system. Due to the deep coupling of the power system, physical space is highly dependent on the cyber space and the reliability of the cyber system also determines the security of the power system at the same time. The CPS characteristic brings the high efficiency, but it also brings a lot of uncertain factors meanwhile. This issue has been confirmed from recent Ukrainian large-scale blackouts [2]: hackers implanted highly destructive malicious software, and the power outages occurred through information attack. Therefore, the reliability assessment of power information and communication network, as an important part of information system, has been put into more and more attention, which is greatly significant to prevent the destruction of the event to occur, ensure safe operation of the power system, and improve the communication department of power communication network management level .

In recent years, in the aspect of reliability analysis for the traditional network or power information communication network, there are some relevant research achievements. For example, the literature [3] proposed the idea and framework of establishing power CPS, and summarized the new features of high coupling of cyber and physics in the reliability analysis of power information and communication network; in the reliability evaluation model of communication network, the [4] modeled and dynamicly analyzed the risk of cross space communication mechanism using cellular automaton theory; literature [5] constructed the matrix model of perplexing relation between topological structure for power communication composite system, which has certain superiority in the real-time vulnerability assessment; [6] constructed a functional failure model for the control and monitoring functions, and analyzed the reliability under the consideration of failure of each kind of function.

In summary, the above-mentioned methods of reliability analysis have their advantages, but they are not very scientific and subjective. This paper adopts the method of factor analysis to analyze index data of power communication network, which can reflect most information of original data to a few variables, resulting in increasing independence between index and reducing the interactivity, 
increasing the scientificity of the evaluation and reducing the subjectivity.

\section{Factor Analysis Correlation Theory}

Factor analysis is also called diathesis analysis, which is a multivariate statistical analysis method to describe the multiple observed variables of a certain thing as a few latent variables [7]. It is assumed to obtain $\mathrm{n}$ sample data, and the original variables are: $x_{1}, x_{2}, \ldots \ldots, x_{\mathrm{m}}$. The original variables can be summed up as the linear combination of $\mathrm{P}$ common factors and special factors. The relationship can be written as Eq. (1):

$$
\begin{aligned}
& x_{1}=a_{11} F_{1}+a_{12} F_{2}+\cdots+a_{1 p} F_{p}+\varepsilon_{1} \\
& x_{2}=a_{21} F_{1}+a_{22} F_{2}+\cdots+a_{2 p} F_{p}+\varepsilon_{2} \\
& \cdots \\
& x_{m}=a_{m 1} F_{1}+a_{m 2} F_{2}+\cdots+a_{m p} F_{p}+\varepsilon_{m}
\end{aligned}
$$

The above mentioned equation is called factors model. Where ' $F_{i}$ ' and ' $\varepsilon_{i}$ ' represent the common factors and specific factors, respectively. ' $a_{i j}$ ' represents the load on the $\mathrm{j}$-th factor of the $\mathrm{i}$-th variable, that is, the correlation coefficient. The larger the absolute value of ' $a_{i j}$ ' is, the greater dependence of both is. The factor load matrix is as follows:

$$
A=\left(a_{i j}\right)_{m \times p}=\left[\begin{array}{cccc}
a_{11} & a_{12} & \cdots & a_{1 p} \\
a_{21} & a_{22} & \cdots & a_{2 p} \\
\cdots & \cdots & \cdots & \cdots \\
a_{m 1} & a_{m 2} & \cdots & a_{m p}
\end{array}\right]
$$

\section{The Reliability Evaluation Model of Power Information Communication Network Based on Factor Analysis}

In the reliability evaluation of power communication network, it is often hoped to collect as many variables as possible to reflect the risk of the power communication network, so as to grasp and understand the problem comprehensively and completely. Although a large sample of multi variable can provide a large amount of information for the risk assessment of power communication network, there is a correlation among many variables in most cases, which means that the massive variables which seem different from each other can not reflect the different attributes of the communication network from each side. On the contrary, they are different forms of expression of a property. The mathematical model of factor analysis can solve the problem effectively.

The brief steps are as follows:

(1) Establish the reliability evaluation index system, that is , determine the variables and collect data.

(2) Standardize data and determine whether the data applies factor analysis.

For the orders of magnitude, the structure and the dimension of the index data of the power communication network is very different, we should carry on the standardization process. There are many methods to determine whether the data is applicable, such as calculating the correlation coefficient matrix, calculating the KMO statistic, and carrying on the Bartlett sphere test. Here, the method of KMO statistics is selected: the method of factor analysis can be freely used when KMO is more than 0.7, it is far-fetched to use factor analysis when KMO is more than 0.6 and less than 0.7 , it is no longer suitable to adopt this method when KMO is less than the above range.

(3) Extract common factors.

The methods can be used for the determination of common factors, such as characteristic value, scree plot, or cumulative contribution rate, and so on. Scree plot is a line graph, whose horizontal coordinates and the longitudinal coordinates represent the serial number of factor and the characteristic value, respectively. The number of common factors is observed easily. When the cumulative contribution rate of variance reaches a certain value, most of the information of the 
original variable can be explained, and the corresponding factor number is the number of the common factor.

(4) Rotate factor axis.

If each column of the data of the initial load matrix do not differ much, it is necessary to rotate the factor axis, that is, to the 0 or 1 poles.

(5) Calculate the common factor score.

Each common factor can be expressed as a linear combination of the reliability evaluation index of the power information communication network:

$$
\begin{aligned}
& F_{1}=\beta_{11} x_{1}+\beta_{12} x_{2}+\cdots+\beta_{1 m} x_{m} \\
& F_{2}=\beta_{21} x_{1}+\beta_{22} x_{2}+\cdots+\beta_{2 p} x_{m} \\
& \cdots \\
& F_{p}=\beta_{m 1} x_{1}+\beta_{m 2} x_{2}+\cdots+\beta_{m p} x_{m}
\end{aligned}
$$

Where ' $\beta_{i j}$ ' represents score coefficient, and ' $x_{i}$ ' stands for indicators data.

(6) Extract the common factor of the variance contribution rate as the weight, combined with the factor scores, and get the comprehensive evaluation of the expression.

$$
F=\frac{b_{1}}{\sum_{i=1}^{p} b_{i}} F_{1}+\frac{b_{2}}{\sum_{i=1}^{p} b_{i}} F_{2}+\cdots+\frac{b_{p}}{\sum_{i=1}^{p} b_{i}} F_{p}
$$

Where ' $F_{i}$ ' and ' $b_{i}$ ' represent the factor scores and variance contribution rate, respectively. 'p' stands for the number of factors.

\section{Example Analysis}

In order to verify the validity of this algorithm, the data of the whole year of a power communication network is selected to evaluate the reliability. The following is the specific evaluation procedures:

Step 1. Construct the index system of reliability evaluation.

Combined with the specific circumstances of the communication network, a reliability evaluation index system is established, as shown in Table1.

Step 2. Standardize data and determine whether the data applies factor analysis.

Calculate the value of $\mathrm{KMO}$ in SPSS: $\mathrm{KMO}=0.781>0.7$. Therefore, it can use the method of factor analysis.

Table 1 The reliability evaluation index system of power information communication network.

\begin{tabular}{ccc}
\hline first-class index & second-class index & Value \\
\hline & SDH security degree & $\geq 0$ \\
communication & PCM security degree & $\geq 0$ \\
device & Carrier device & $\geq 0$ \\
& Switch & $\geq 0$ \\
Power Supply & $\geq 0$ \\
operating & thunder & $1 \sim 5(5$ level $)$ \\
environment & Ice and snow & $\geq 0$ \\
& wind & $1 \sim 5(5$ level $)$ \\
optical fibre & temperature & $-52.3 \sim 50^{\circ} \mathrm{C}$ \\
& other & $\geq 0$ \\
business & time delay & $>5 \mu \mathrm{s}$ \\
& Intrinsic loss & $0.305 \sim 0.320 \mathrm{~dB} / \mathrm{km}$ \\
peration and & splice loss & $0.50 \sim 100 \mathrm{~dB} / \mathrm{km}$ \\
maintenance & Business critical level & $\geq 0$ \\
& average outage time & $\geq 0$ \\
& plan completion rate & $\geq 0$ \\
& Maintenance condition & $\geq 0$ \\
& Personnel management & $\geq 0$ \\
\hline
\end{tabular}


Step 3. Extract common factors.

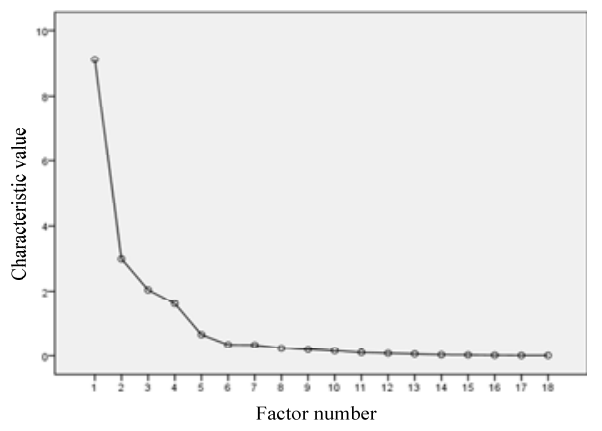

Fig. 1. Scree plot

By observing the scree plot, shown in Figure 1, the first four factors in the "steep" may be determined as common factors. The characteristic values of factors are small after the slope slowing down, which can not be considered.

From the point of the cumulative variance, the first four factors can explain the $87.472 \%$ of all the indicator information, then taking the 4 factor as the common factors is reasonable. As shown in the Table 2.

Table 2 The total variance decomposition table

\begin{tabular}{|c|c|c|c|c|c|c|c|c|c|}
\hline \multirow{2}{*}{ component } & \multicolumn{3}{|c|}{ Initial Eigenvalues } & \multicolumn{3}{|c|}{$\begin{array}{c}\text { Extraction Sums of Squared } \\
\text { Loadings }\end{array}$} & \multicolumn{3}{|c|}{$\begin{array}{l}\text { Rotation Sums of } \\
\text { Squared Loadings }\end{array}$} \\
\hline & Total & $\begin{array}{c}\% \text { of } \\
\text { Variance }\end{array}$ & $\begin{array}{c}\text { Cumulative } \\
\%\end{array}$ & Total & $\begin{array}{c}\% \text { of } \\
\text { Variance }\end{array}$ & $\begin{array}{c}\text { Cumulative } \\
\%\end{array}$ & Total & $\begin{array}{c}\% \text { of } \\
\text { Variance }\end{array}$ & $\begin{array}{c}\text { Cumulative } \\
\%\end{array}$ \\
\hline 1 & 9.102 & 50.566 & 50.566 & 9.102 & 50.566 & 50.566 & 8.705 & 48.360 & 48.360 \\
\hline 2 & 2.989 & 16.606 & 67.171 & 2.989 & 16.606 & 67.171 & 2.739 & 15.218 & 63.578 \\
\hline 3 & 2.043 & 11.352 & 78.523 & 2.043 & 11.352 & 78.523 & 2.274 & 12.631 & 76.209 \\
\hline 4 & 1.611 & 8.949 & 87.472 & 1.611 & 8.949 & 87.472 & 2.027 & 11.263 & 87.472 \\
\hline 5 & 0.659 & 3.659 & 91.131 & & & & & & \\
\hline 6 & 0.343 & 1.904 & 93.036 & & & & & & \\
\hline 7 & 0.335 & 1.860 & 94.895 & & & & & & \\
\hline 8 & 0.234 & 1.298 & 96.193 & & & & & & \\
\hline 9 & 0.198 & 1.101 & 97.295 & & & & & & \\
\hline 10 & 0.156 & 0.868 & 98.162 & & & & & & \\
\hline 11 & 0.102 & 0.566 & 98.728 & & & & & & \\
\hline 12 & 0.078 & 0.435 & 99.163 & & & & & & \\
\hline 13 & 0.058 & 0.323 & 99.486 & & & & & & \\
\hline 14 & 0.032 & 0.178 & 99.664 & & & & & & \\
\hline 15 & 0.026 & 0.147 & 99.811 & & & & & & \\
\hline 16 & 0.015 & 0.085 & 99.896 & & & & & & \\
\hline 17 & 0.010 & 0.058 & 99.953 & & & & & & \\
\hline 18 & 0.008 & 0.047 & 100.000 & & & & & & \\
\hline
\end{tabular}

Step 4. Rotate factor axis.

The data of the initial load matrix do not differ much, so it need to rotate the factor axis. After the rotation of the factor load matrix, as shown in Table 3.

Table 3 Rotated component matrix

\begin{tabular}{|c|c|c|c|c|c|c|c|c|c|}
\hline \multirow{2}{*}{ index } & \multicolumn{4}{|c|}{ component } & \multirow{2}{*}{ index } & \multicolumn{4}{|c|}{ component } \\
\hline & 1 & 2 & 3 & 4 & & 1 & 2 & 3 & 4 \\
\hline SDH security degree & .926 & -.237 & .160 & .077 & other & .770 & .215 & .275 & -.392 \\
\hline PCM security degree & .917 & -.114 & .151 & .201 & time delay & .616 & .328 & .387 & .267 \\
\hline carrier device & .904 & .133 & -.185 & .225 & intrinsic loss & .961 & .110 & .140 & -.023 \\
\hline switch & .903 & .131 & -.105 & .087 & splice loss & .936 & .179 & .049 & -.128 \\
\hline power supply & .701 & .080 & -.561 & .091 & business critical level & .089 & -.037 & -.028 & -.920 \\
\hline thunder & .331 & .912 & -.075 & .013 & average outage time & .685 & .515 & -.109 & .200 \\
\hline ice and snow & .449 & -.837 & .193 & .048 & $\begin{array}{l}\text { operational plan } \\
\text { completion rate }\end{array}$ & .568 & .245 & -.171 & .622 \\
\hline wind & .146 & -.250 & .766 & -.323 & maintenance condition & .271 & .709 & .208 & .493 \\
\hline temperature & .948 & .060 & .164 & -.078 & personnel management & .075 & .065 & .936 & .208 \\
\hline
\end{tabular}

Step 5. Compute factor scores using the formula (2) .

Make use of spss to calculate the score coefficient matrix firstly, as shown in Table 4. and then substitute into the formula (2) to obtain common factor score. 
Table 4 Score coefficient matrix

\begin{tabular}{|c|c|c|c|c|c|c|c|c|c|}
\hline \multirow{2}{*}{ index } & \multicolumn{4}{|c|}{ score coefficient } & \multirow{2}{*}{ index } & \multicolumn{4}{|c|}{ score coefficient } \\
\hline & 1 & 2 & 3 & 4 & & 1 & 2 & 3 & 4 \\
\hline SDH security degree & .122 & -.150 & .028 & .043 & other & .090 & .111 & .095 & -.260 \\
\hline PCM security degree & .111 & -.113 & .034 & .097 & time delay & .038 & .093 & .176 & .097 \\
\hline carrier device & .108 & -.027 & -.106 & .071 & intrinsic loss & .113 & .005 & .032 & -.055 \\
\hline switch & .109 & -.007 & -.073 & -.002 & splice loss & .113 & .044 & -.008 & -.122 \\
\hline power supply & .103 & -.041 & -.276 & .001 & business critical level & .046 & .088 & -.043 & -.504 \\
\hline thunder & -.003 & .363 & -.002 & -.115 & average outage time & .058 & .153 & -.045 & .022 \\
\hline ice and snow & .093 & -.374 & .032 & .116 & $\begin{array}{l}\text { operational plan } \\
\text { completion rate }\end{array}$ & .045 & -.009 & -.070 & .288 \\
\hline wind & .014 & -.038 & .322 & -.131 & maintenance condition & -.027 & .236 & .132 & .183 \\
\hline temperature & .115 & -.007 & .040 & -.078 & personnel management & -.036 & .044 & .432 & .129 \\
\hline
\end{tabular}

Data were collected for 48 times in the whole year, with an average of 4 times per month. Fig. 2 is the visual display of the value of the four common factors.

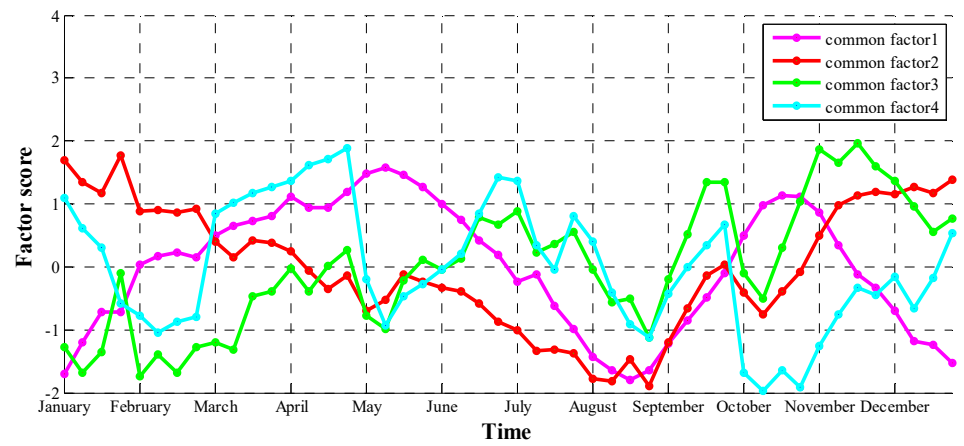

Fig. 2. The value of the four common factors

Step 6. Use formula (3) to draw the comprehensive evaluation results.

Combined with factor scores, take cumulative variance contribution rate as the respective weights and substitute into the formula (3) to find out the value of evaluation.

Taking a collection of data for example: factor score is $-1.69525,1.69435,-1.27024$ and 1.10486 . The weights of the four factors are: $50.566,16.606,11.352$ and 8.949, into formula(3):

$$
\begin{aligned}
F & =\frac{-1.69525 \times 50.566+1.69435 \times 16.606}{50.566+16.606+11.352+8.949}+\frac{(-1.27024) \times 11.352+1.10486 \times 8.949}{50.566+16.606+11.352+8.949} \\
& \approx-0.71
\end{aligned}
$$

Similarly, obtain quantitative assessment results for other months. Finally, visualize its reliability trends:

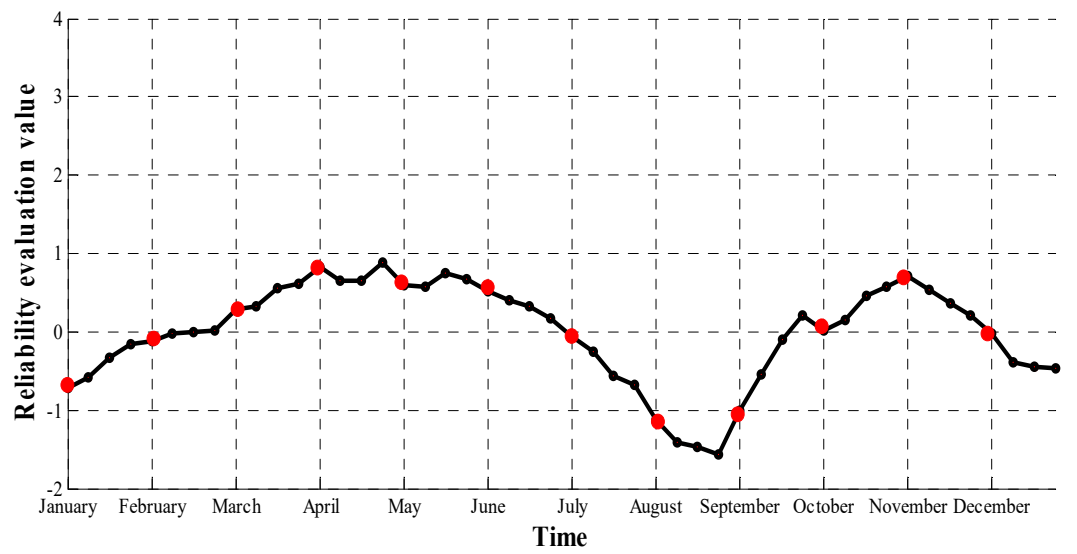

Fig. 3 The reliability trend in the whole year

As shown in Fig 3, the trend of reliability fluctuates over time, and it is low in January, July, August, and December, which may be associated with the summer lightning, winter snow and ice. It is necessary to adjust the defensive measures during the period and improve the reliability. 


\section{Conclusion}

For the reliability assessment of power information communication network is a multi index issue where the indexes have deep dependence and coupling, a comprehensive reliability evaluation model is constructed. The simulation results show that the model can effectively and objectively analyze the overall trend of the risks of power communication network and make predictions. It effectively avoids the defects of the subjective weighting, and solves the issue of the correlation and repeatability between indicators, which has certain practical value.

\section{References}

[1] ZHAO Junhua, WEN Fushuan, XUE Yusheng, et al. Modeling analysis and control research framework of cyber physical power systems[J]. Automation of Electric Power Systems, 2011, 35(16): 1-8.

[2] GUO Qinglai, XIN Shujun, WANG Jianhui, et al. Comprehensive security assessment for a cyber physical energy system: a Lesson: from Ukraine's blackout[J]. Automation of Electric Power Systems, 2016, 40(5): 145-147.

[3] ZHAO Junhua, WEN Fushuan, XUE Yusheng, et al. Cyber physical power systems: architecture, implementation techniques and challenges[J]. Automation of Electric Power Systems, 2010, 34(16): 1-7.

[4] YE Xiaming, WEN Fushuan, SHANG Jincheng, et al. Propagation mechanism of cyber physical security risks in power systems[J]. Power System Technology, 2015, 39(11): 3072- 3079.

[5] TANG Yi, HAN Xiao, WU Yingjun, et al. Electric Power System Vulnerability Assessment Considering the Influence of Communication System[J]. Proceedings of the CSEE, 2015, 35(23): 6066-6074.

[6] GUO Jia, HAN Yuqi, GUO Chuangxin, et al. Reliability assessment of cyber physical power system considering monitoring function and control function[J]. Proceedings of the CSEE, 2016, 36(8): 2123-2130.

[7] DU Zhimin, FAN Wenqiang. The application of SPSS in social survey[M]. Beijing: electronics industry press, 2015: 413-437. 